\title{
A Mixed Finite-Element Discretization of the Energy-Transport Model for Semiconductors
}

\author{
Stefan Holst \\ Ansgar Jüngel \\ Paola Pietra
}

Konstanzer Schriften in Mathematik und Informatik

Nr. 158, Oktober 2001

ISSN 1430-3558

Konstanzer Online-Publikations-System (KOPS)

URL: http://www.ub.uni-konstanz.de/kops/volltexte/2006/2181/

URN: http://nbn-resolving.de/urn:nbn:de:bsz:352-opus-21815

(C) Fachbereich Mathematik und Statistik

(C) Fachbereich Informatik und Informationswissenschaft

Universität Konstanz

Fach D 188, 78457 Konstanz, Germany

Email: preprints@informatik.uni-konstanz.de

WWW: http://www.informatik.uni-konstanz.de/Schriften/ 


\title{
A Mixed Finite-Element Discretization of the Energy-Transport Model for Semiconductors
}

\author{
Stefan Holst, Ansgar Jüngel \\ Fachbereich Mathematik und Statistik, Fach D193, \\ 78457 Konstanz, Universität Konstanz, Germany, \\ e-mail: stefan.holst@uni-konstanz.de, \\ ansgar . juengel@uni-konstanz.de. \\ Paola Pietra \\ Istituto di Analisi Numerica, C.N.R., \\ Via Ferrata 1, 27100 Pavia, Italy, \\ e-mail: pietra@dragon.ian.pv.cnr.it.
}

\begin{abstract}
Energy-transport models describe the flow of electrons through a semiconductor device, influenced by diffusive, electrical, and thermal effects. They consist of the continuity equations for the mass and energy, coupled with Poisson's equation for the electrostatic potential. The energy-transport model can be written in a driftdiffusion formulation which is used for the numerical approximation. The stationary equations are discretized with an exponential fitting mixed finite-element method in two space dimensions. Numerical simulations of a ballistic diode are performed and numerical convergence rates are computed. Furthermore, a two-dimensional MESFET device with parabolic band structure is simulated.
\end{abstract}

Key words. Mixed finite elements, exponential fitting, two-dimensional MESFET, semiconductors.

AMS Subject classification. 65N30, 82D55, 78A35.

Acknowledgments. The authors acknowledge partial support from the TMR Project "Asymptotic Methods in Kinetic Theory", grant ERB-FMBX-CT97-0157. The first and second author are partly supported by the Gerhard-Hess Programm of the Deutsche Forschungsgemeinschaft, grant JU395/3, and by the AFF Project of the University of Konstanz, grant 4/00.

\section{Introduction}

In the modeling of semiconductor devices, two main classes of classical models can be distinguished: kinetic models, like the semiconductor Boltzmann equation, and fluid-dynamical models, like the drift-diffusion equations [21, 25, 31]. The semiconductor Boltzmann equation gives quite accurate simulation results, 
but the numerical methods to solve this equation (for instance, with MonteCarlo methods [40]) are too costly and time consuming to model real problems in semiconductor production mode where simulation results are needed in hours or minutes. Acceptable accuracy can be obtained by solving macroscopic (fluid-dynamical) model equations derived from the Boltzmann equation. The simplest model are the drift-diffusion equations for which very efficient numerical algorithms are available $[6,20,22,26]$. These models, however, are not accurate enough for sub-micron device modeling, owing to temperature effects of hot electrons, for instance.

The energy-transport equations are able to model temperature effects. They are of parabolic type such that their numerical solution needs less effort than hydrodynamic models which contain hyperbolic modes $[4,9,17]$. Moreover, the energy-transport models we study here can be written in a drift-diffusion form. Therefore, the numerical effort is comparable to the drift-diffusion equations and numerical schemes, developed for the drift-diffusion models, can be employed.

In this paper an exponential fitting mixed finite-element method is used to discretize the stationary energy-transport equations in two space dimensions. More precisely, we adopt the finite-element scheme, proposed by Marini and Pietra in $[29,30]$ for the drift-diffusion equations, to the energy-transport system.

The energy-transport equations consist of the conservation laws of mass and energy, together with constitutive relations for the particle and energy currents, and are coupled to the Poisson equation for the electrostatic potential. Denoting by $\mu, T$, and $V$ the chemical potential, the electron temperature, and the electrostatic potential, and by $J_{1}, J_{2}$ the particle and the energy current densities, respectively, the stationary equations in the entropic variables $\mu / T$ and $-1 / T$ read as follows:

$$
\begin{aligned}
-\operatorname{div} J_{1} & =0, \\
-\operatorname{div} J_{2} & =-J_{1} \cdot \nabla V+W(n, T), \\
J_{1} & =L_{11}\left(\nabla \frac{q \mu}{k_{B} T}-\frac{q \nabla V}{k_{B} T}\right)+L_{12} \nabla\left(-\frac{1}{k_{B} T}\right), \\
q J_{2} & =L_{21}\left(\nabla \frac{q \mu}{k_{B} T}-\frac{q \nabla V}{k_{B} T}\right)+L_{22} \nabla\left(-\frac{1}{k_{B} T}\right), \\
\varepsilon_{s} \Delta V & =q(n-C) .
\end{aligned}
$$

The physical constants are the elementary charge $q$, the Boltzmann constant $k_{B}$, and the semiconductor permittivity $\varepsilon_{s}$. The electron density $n$ depends on $\mu$ and $T$. The space dependent function $C=C(x)$ is the doping profile, $L_{i j}=L_{i j}(n, T)$ are the diffusion coefficients, and $W=W(n, T)$ is the energy relaxation term [25]. The above equations have to be solved in the bounded semiconductor domain $\Omega \subset \mathbb{R}^{d}$. We complement the equations with physically motivated mixed Dirichlet-Neumann boundary conditions:

$$
\begin{aligned}
n=n_{D}, \quad T=T_{D}, \quad V=V_{D} & \text { on } \Gamma_{D}, \\
J_{1} \cdot \nu=J_{2} \cdot \nu=\nabla V \cdot \nu=0 & \text { on } \Gamma_{N},
\end{aligned}
$$


modeling the contacts $\Gamma_{D}$ and the insulating boundary parts $\Gamma_{N}$. We have assumed that $\partial \Omega=\Gamma_{D} \cup \Gamma_{N}$ and $\Gamma_{D} \cap \Gamma_{N}=\emptyset$. The exterior normal unit vector on $\partial \Omega$ is denoted by $\nu$.

The system (1)-(5) has been studied analytically in [11, 12] under the assumption of uniformly bounded diffusion coefficients. The existence and uniqueness of weak solutions to both the stationary and the time-dependent (initial) boundary-value problems have been proved. Existence results with different assumptions (for instance, near-equilibrium situations) have been shown in $[1,15,19,24]$.

The first energy-transport model has been presented by Stratton in 1962 [37]. In the physical literature, energy-transport equations have been derived from hydrodynamic models usually by neglecting certain convection terms (see, e.g., [34] and references therein). This approach can be made rigorously by considering a diffusion time scaling [18]. Another approach is to derive the energytransport equations from the Boltzmann equation by means of the Hilbert expansion method [3]. First, a so-called spherical harmonic expansion (SHE) model is derived from the Boltzmann equation in the diffusion limit, under the assumption of dominant elastic scattering. Then, through a diffusion approximation, respectively making electron-electron or phonon scattering large, the energy-transport equations are derived from the SHE model. The advantage of this approach is that it can be performed under quite weak assumptions on the semiconductor band structure and that explicit expressions for the diffusion coefficients and the energy relaxation term can be given [13]. Moreover, any energy-transport model derived in this way (assuming Boltzmann statistics) allows for a drift-diffusion formulation of the form

$$
J_{i}=\nabla g_{i}(n, T)-g_{i}(n, T) \frac{\nabla V}{T}, \quad i=1,2,
$$

where $g_{1}$ and $g_{2}$ are nonlinear functions of $n$ and $T$. (In fact, $g_{1}=L_{11}$ and $g_{2}=L_{21}$; see [13] for details.) This formulation is the basis for our numerical discretization. Notice that for constant temperature, this expression reduces to the standard drift-diffusion current definition.

In the physical literature, the energy-transport equations have been investigated numerically for several years [2, 7, 8, 36, 39], usually using ScharfetterGummel-type discretizations. Jerome and Shu [23] solved the equations employing ENO (essentially non-oscillatory) methods. Compact finite-difference schemes have been used in [16]. The energy-transport model in the dual entropy formulation has been discretized with mixed finite elements by Marrocco et al. [32]. Another mixed finite-element discretization has been employed in [13], but only in one space dimension.

The continuity equations (1), (2) and (6) are discretized in this paper in two space dimensions with a variant of the mixed exponential fitting scheme, which has been developed and studied in $[6,30]$ for the linear drift-diffusion equations and extended to a nonlinear drift-diffusion model in [26]. The most important features of this scheme are the current conservation (the current is introduced as an independent variable and continuity is directly imposed) and the ability 
to approximate accurately steep gradients.

Since the energy relaxation term can be written in the form $W=c_{1} g_{1}-c_{2} g_{2}$ with $c_{i}=c_{i}\left(g_{1}, g_{2}\right) \geq 0$ (see Section 2), we can write the continuity equation $(2),(6)$ in the variables $g_{1}$ and $g_{2}$ :

$$
-\operatorname{div} J_{2}+c_{2} g_{2}=f, \quad J_{2}=\nabla g_{2}-g_{2} \frac{\nabla V}{T\left(g_{1}, g_{2}\right)},
$$

where $f$ contains the Joule heating term $J_{1} \cdot \nabla V$ and the term $c_{1} g_{1}$. For the case $c_{2}=0$ and constant temperature, a mixed scheme, based on the lowestorder Raviart-Thomas elements [33], has been introduced and discussed in [6] for $f=0$ and in [5] for $f \neq 0$. The matrix associated with the scheme can be proved to be an M-matrix, if a weakly acute triangulation is used. This property guarantees a discrete maximum principle and, in particular, a nonnegative solution if the boundary data are non-negative. Unfortunately, the M-matrix property does not hold anymore if $c_{2} \neq 0$. In order to circumvent this fact we use the finite elements developed and analyzed by Marini and Pietra $[29,30]$. In fact, it has been proved that these elements provide an M-matrix for all $c_{2} \geq 0$.

This paper is organized as follows. In Section 2 we scale the equations and make explicit the model assumptions. In particular, we assume parabolic band diagrams (see [13] for nonparabolic bands). The mixed finite-element discretization using the elements of Marini and Pietra is derived and the global iteration procedure is explained in Section 3. Section 4 is devoted to the application of the resulting scheme to a two-dimensional ballistic diode, which is uniform in one space dimension (for comparison with the results in [13]) and to a twodimensional MESFET device. The paper finishes with a short conclusion in Section 5 .

\section{The Model Assumptions}

In this section we scale the equations (1)-(5) appropriately and specify our assumptions on the constitutive relations for $L_{i j}, n$ and $W$ (or $g_{1}, g_{2}$ and $W$ ).

Let $C_{m}$ be the maximal value of the doping profile, $\ell^{*}$ the diameter of the device, $\mu_{0}$ the low-field mobility, $T_{0}$ the lattice temperature, and $U_{T}=k_{B} T_{0} / q$ the thermal voltage. Using the scaling

$$
\begin{gathered}
n \rightarrow C_{m} n, \quad C \rightarrow C_{m} C, \quad T \rightarrow T_{0} T, \quad \mu \rightarrow U_{T} \mu, \quad x \rightarrow \ell^{*} x, \\
J_{1} \rightarrow\left(q \mu_{0} U_{T} C_{m} / \ell^{*}\right) J_{1}, \quad J_{2} \rightarrow\left(q \mu_{0} U_{T}^{2} C_{m} / \ell^{*}\right) J_{2}, \\
L_{i j} \rightarrow\left(\left(q U_{T}\right)^{i+j-1} \mu_{0} C_{m}\right) L_{i j}, \quad W \rightarrow\left(q \mu_{0} U_{T}^{2} C_{m} / \ell^{* 2}\right) W
\end{gathered}
$$


we obtain the system

$$
\begin{aligned}
-\operatorname{div} J_{1} & =0, \\
-\operatorname{div} J_{2} & =-J_{1} \cdot \nabla V+W(n, T), \\
J_{1} & =L_{11}\left(\nabla \frac{\mu}{T}-\frac{\nabla V}{T}\right)+L_{12} \nabla\left(-\frac{1}{T}\right), \\
J_{2} & =L_{21}\left(\nabla \frac{\mu}{T}-\frac{\nabla V}{T}\right)+L_{22} \nabla\left(-\frac{1}{T}\right), \\
\lambda^{2} \Delta V & =n-C,
\end{aligned}
$$

where $\lambda=\sqrt{\varepsilon_{s} U_{T} /\left(q C_{m} \ell^{* 2}\right)}$ is the scaled Debye length.

We impose the following physical assumptions:

- The energy-band diagram is parabolic.

- The electron density is given by nondegenerate Boltzmann statistics.

- The energy relaxation term is given by a Fokker-Planck approximation (see [13, section 2.2]).

Under these hypotheses it is shown in [13] that the current densities (9)-(10) can be written in the form (6) and that the following relations hold:

$$
\begin{aligned}
n & =N_{c} T^{3 / 2} \exp (\mu / T), \quad \text { where } N_{c}>0, \\
g_{i}(n, T) & =\mu_{0} \frac{2 \Gamma(i+1-\beta)}{\sqrt{\pi}} T^{i-1 / 2-\beta} n, \quad i=1,2, \\
W(n, T) & =-\frac{3}{2} \frac{n\left(T-T_{0}\right)}{\tau_{\beta}(T)}, \\
\tau_{\beta}(T) & =\tau_{0} \frac{3 \sqrt{\pi}}{4 \Gamma(\beta+2)} T^{1 / 2-\beta} .
\end{aligned}
$$

Here, $\tau_{\beta}(T)$ is the energy relaxation time, and the constant $\beta>-2$ is a parameter in the energy relaxation model. For the precise definition of the parameters $N_{i}, \tau_{0}$ and $\mu_{0}$ we refer to [13, section 2.4]. The symbol $\Gamma$ denotes the Gamma function defined by

$$
\Gamma(s)=\int_{0}^{\infty} u^{s-1} e^{-u} d u, \quad s>0 .
$$

In the literature, the values $\beta=1 / 2$ (used by Chen et al. [7]) and $\beta=0$ (used by Lyumkis et al. [27]) have been employed. In the case $\beta=1 / 2$ we have $\Gamma(3 / 2)=\sqrt{\pi} / 2$ and $\Gamma(5 / 2)=3 \sqrt{\pi} / 4$ and therefore, since $T_{0}=1$,

$$
\begin{gathered}
g_{1}(n, T)=\mu_{0} n, \quad g_{2}(n, T)=\frac{3}{2} \mu_{0} n T, \\
W=-\frac{3}{2} \frac{n\left(T-T_{0}\right)}{\tau_{0}}=\frac{1}{\tau_{0} \mu_{0}}\left(\frac{3}{2} g_{1}-g_{2}\right) .
\end{gathered}
$$


We call the energy-transport equations with these relations for $g_{1}, g_{2}$ and $W$ the Chen model.

If $\beta=0$ we obtain

$$
\begin{aligned}
& g_{1}(n, T)=\frac{2}{\sqrt{\pi}} \mu_{0} n T^{1 / 2}, \quad g_{2}(n, T)=\frac{4}{\sqrt{\pi}} \mu_{0} n T^{3 / 2}, \\
& W=-\frac{2}{\sqrt{\pi}} \frac{n\left(T-T_{0}\right)}{\sqrt{T}}=\frac{1}{\tau_{0} \mu_{0} T\left(g_{1}, g_{2}\right)}\left(g_{1}-\frac{1}{2} g_{2}\right) .
\end{aligned}
$$

The function $T\left(g_{1}, g_{2}\right)$ equals $g_{2} /\left(2 g_{1}\right)$. The corresponding energy-transport equations are called the Lyumkis model. We use the above formulation of $W\left(g_{1}, g_{2}\right)$ including $T\left(g_{1}, g_{2}\right)$ since this allows us to write the energy equation in the form

$$
\operatorname{div} J_{2}+c_{2} g_{2}=f
$$

with $c_{2}=1 /\left(2 \tau_{0} \mu_{0} T\right)$ (see Section 1 ).

We rewrite the energy-transport model in the $\left(g_{1}, g_{2}, V\right)$ variables:

$$
\begin{aligned}
& -\operatorname{div} J_{1}=0, \\
& -\operatorname{div} J_{2}+c_{2}\left(g_{1}, g_{2}\right) g_{2}=c_{1}\left(g_{1}, g_{2}\right) g_{1}-J_{1} \cdot \nabla V, \\
& J_{i}=\nabla g_{i}-g_{i} \frac{\nabla V}{T\left(g_{1}, g_{2}\right)}, \quad i=1,2, \\
& \lambda^{2} \Delta V=n\left(g_{1}, g_{2}\right)-C(x) \quad \text { in } \Omega, \\
& g_{1}=g_{D, 1}, \quad g_{2}=g_{D, 2}, \quad V=V_{D} \quad \text { on } \Gamma_{D}, \\
& J_{1} \cdot \nu=J_{2} \cdot \nu=\nabla V \cdot \nu=0 \quad \text { on } \Gamma_{N},
\end{aligned}
$$

where $g_{D, i}=g_{i}\left(n_{D}, T_{D}\right), i=1,2$, and

$$
\begin{aligned}
\text { Chen: } \quad c_{1}\left(g_{1}, g_{2}\right) & =\frac{3}{2 \tau_{0} \mu_{0}}, \quad c_{2}\left(g_{1}, g_{2}\right)=\frac{1}{\tau_{0} \mu_{0}}, \\
T\left(g_{1}, g_{2}\right) & =\frac{2 g_{2}}{3 g_{1}}, \quad n\left(g_{1}, g_{2}\right)=\frac{g_{1}}{\mu_{0}}, \\
\text { Lyumkis: } \quad c_{1}\left(g_{1}, g_{2}\right) & =\frac{1}{\tau_{0} \mu_{0} T\left(g_{1}, g_{2}\right)}, \quad c_{2}\left(g_{1}, g_{2}\right)=\frac{1}{2 \tau_{0} \mu_{0} T\left(g_{1}, g_{2}\right)}, \\
T\left(g_{1}, g_{2}\right) & =\frac{g_{2}}{2 g_{1}}, \quad n\left(g_{1}, g_{2}\right)=\left(\frac{\pi}{2 \mu_{0}^{2}} \frac{g_{1}^{3}}{g_{2}}\right)^{1 / 2} .
\end{aligned}
$$

\section{The Numerical Scheme}

\subsection{Mixed Finite-Element Discretization}

In this section we describe the discretization of the energy-flux equations (13), (14) using an exponential fitting mixed finite-element method. The equations (12), (14) are treated similarly. In fact, their discretization is simpler since 
the right-hand side of (12) vanishes. For the Poisson equation (15), $P_{1}$ nonconforming elements are used (cf. [10]). Therefore, in the forthcoming, we assume $V$ to be linear on each triangle.

Let $\Omega \subset \mathbb{R}^{2}$ be a polygonal domain and let $\mathcal{T}_{h}$ be a regular family of decompositions of $\Omega$ into triangles $K$ in such a way that there is no element across the interface between $\Gamma_{D}$ and $\Gamma_{N}$. Furthermore, let $\mathcal{E}_{h}$ be the set of edges $e$ of $\mathcal{T}_{h}$. As in $[13,26]$ the idea of the discretization is to transform the problem by means of the Slotboom variable to a symmetric form, then to discretize the symmetric form with mixed finite elements (consequently, the flux is introduced as independent variable), and to use a suitable discrete change of variable to return to the original unknown $g_{2}$. Due to the nonconstant electron temperature, a global Slotboom variable does not exist. However, we can define a "local" Slotboom variable $y_{2}$, assuming that the temperature $T=T\left(g_{1}, g_{2}\right)$ is a prescribed piecewise constant function, called $\bar{T}$, defined in the global iteration process. Therefore, $\nabla V / \bar{T}$ is constant on each element $K$, and we can define

$$
y_{2}=e^{-V / \bar{T}} g_{2} \text { in } K
$$

under the assumption $\left.\bar{T}\right|_{K}>0$. Equations (13) and (14) can be rewritten on each triangle $K$ with $\bar{c}_{i}=c_{i}(\bar{T})$ (see (16) or (18)) as follows:

$$
\begin{aligned}
e^{-V / \bar{T}} J_{2}-\nabla y_{2} & =0, \\
-\operatorname{div} J_{2}+\bar{c}_{2} e^{V / \bar{T}} y_{2} & =-J_{1} \cdot \nabla V+\bar{c}_{1} g_{1} \quad \text { in } \Omega .
\end{aligned}
$$

To derive the monotone mixed scheme developed in [30], a special set of polynomial vectors are introduced to approximate the current density:

$$
\Sigma(K)=\operatorname{span}\left(\tau_{1}, \tau_{2}, \tau_{3}\right),
$$

with

$$
\tau_{1}=(1,0), \quad \tau_{2}=(0,1), \quad \tau_{3}=\left(\omega_{1}, \omega_{2}\right) .
$$

To define the properties that determine the polynomials $\omega_{i}$ we need the following notation. The edges of each triangle $K$ are numbered counter-clockwise starting with $e_{1}$, which is the edge connecting the vertices with the smallest values of $V$ in $K$ (see Figure 1 ). We choose $\tau_{3}=\left(\omega_{1}, \omega_{2}\right)$ with $\omega_{1}, \omega_{2} \in P_{2}(K)$ fulfilling the following conditions:

$$
\begin{gathered}
\left.\tau_{3} \cdot \nu_{2}\right|_{e_{2}}=\left.\tau_{3} \cdot \nu_{3}\right|_{e_{3}}=0,\left.\quad \tau_{3} \cdot \nu_{1}\right|_{e_{1}}=1, \\
\int_{K} \tau_{3} \cdot \operatorname{curl}\left(\lambda_{1}, \lambda_{2}, \lambda_{3}\right) d x d y=0, \\
\int_{K} \omega_{1} d x d y=\int_{K} \omega_{2} d x d y=0,
\end{gathered}
$$

where $\lambda_{i}$ denotes the $i$-th barycentric coordinate. It is easily checked that $\operatorname{dim}(\operatorname{div} \Sigma(K))=1($ see $(23))$. 


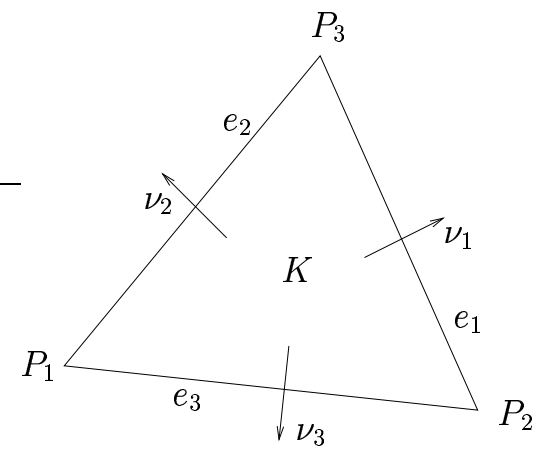

Figure 1: Notation in a triangle $K$.

We introduce the following finite dimensional spaces:

$$
\begin{aligned}
V_{h} & =\left\{\tau \in\left[L^{2}(\Omega)\right]^{2}:\left.\tau\right|_{K} \in \Sigma(K) \forall K \in \mathcal{T}_{h}\right\}, \\
W_{h} & =\left\{\phi \in L^{2}(\Omega):\left.\phi\right|_{K} \in P_{0}(K) \forall K \in \mathcal{T}_{h}\right\}, \\
\Lambda_{h, \xi} & =\left\{\mu \in L^{2}\left(\mathcal{E}_{h}\right):\left.\mu\right|_{K} \in P_{0}(e) \forall e \in \mathcal{E}_{h} ; \int_{e}(\mu-\xi) d s=0 \forall e \in \Gamma_{D}\right\},
\end{aligned}
$$

where $\xi$ is any function in $L^{2}\left(\Gamma_{D}\right)$. The spaces $P_{0}(K)$ and $P_{0}(e)$ denote the sets of constant functions in $K$ and on $e$, respectively. The mixed-hybrid formulation of (21)-(22) is:

$$
\left\{\begin{array}{l}
\text { Find } J_{2}^{h} \in V_{h}, \bar{g}_{2}^{h} \in W_{h} \text { and } g_{2}^{h} \in \Lambda_{h, g_{D, 2}} \text { such that: } \\
\sum_{K \in \mathcal{T}_{h}}\left(\int_{K} R_{K} J_{2}^{h} \cdot \tau d x+\int_{K} S_{K} \bar{g}_{2}^{h} \operatorname{div} \tau d x-\sum_{e_{i}(K)} \int_{e_{i}} S_{e_{i}} g_{2}^{h} \tau \cdot \nu d s\right)=0, \\
\sum_{K \in \mathcal{T}_{h}}\left(-\int_{K} \operatorname{div} J_{2}^{h} \phi d x+\int_{K} \bar{c}_{2} \bar{g}_{2}^{h} \phi d x\right)=\int_{K}\left(-J_{1}^{h} \cdot \nabla V+\bar{c}_{1} \bar{g}_{1}^{h}\right) \phi d x, \\
\sum_{K \in \mathcal{T}_{h}} \int_{\partial K} \mu J_{2}^{h} \cdot \nu d s=0
\end{array}\right.
$$

for all $\tau \in V_{h}, \phi \in W_{h}$, and $\mu \in \Lambda_{h, 0}$. Here, $\nu$ denotes the normal unit vector on $\partial K$ and $e_{i}(K)$ is the set of edges $e_{i}$ of $K$. Furthermore, $J_{1}^{h} \in V_{h}$ is the approximation of the current density $J_{1}$ and $\bar{g}_{1} \in W_{h}$ is a piecewise constant approximation of $g_{1}$. The first equation in (27) is a weak discrete version of (21), the second equation corresponds to a discrete version of (22), and the third equation imposes a continuity requirement of the normal component of $J_{2}^{h}$ at the interelement boundaries. At first the equation states that the jump of $J_{2}^{h} \cdot \nu$ has zero mean value since $\mu$ is constant on each edge. But since $J_{2}^{h}$ itself is constant on any edge (see (23)), the normal component of $J_{2}^{h}$ is continuous across the interelement boundaries. 
The function $R$ denotes a piecewise constant function that is defined on each triangle $K$ by

$$
R_{K}=\frac{1}{|K|} \int_{K} e^{-V / \bar{T}} d x
$$

The piecewise constant function $S$ is defined via an average on the edges. The goal is to approximate large gradients of the potential with the correct order of magnitude (for a detailed discussion see [30, section 3]). More precisely, $S=S_{K}$ on $K$ is given by:

$$
S_{e_{i}(K)}=\frac{1}{\left|e_{i}\right|} \int_{e_{i}} e^{-V / \bar{T}} d s, \quad S_{K}=\max _{i} S_{e_{i}(K)}=S_{e_{1}(K)} .
$$

Recall that the edge $e_{1}$ connects the vertices with the smallest values of $V$ in $K$. The terms $S_{K} \bar{g}_{2}^{h}$ and $S_{e_{i}} g_{2}^{h}$ are both approximations of $y_{2}$.

We describe now the static condensation procedure that eliminates $J_{2}^{h}$ and $\bar{g}_{2}^{h}$ and leads to the final algebraic system in the variable $g_{2}^{h}$. Denoting by $J_{2}^{h}$, $\bar{g}_{2}^{h}$, and $g_{2}^{h}$ the vectors of the nodal values, the linear system associated with (27) then reads as follows:

$$
\left(\begin{array}{rrr}
A & \widetilde{B} & -\widetilde{C} \\
B^{T} & -D & 0 \\
-C^{T} & 0 & 0
\end{array}\right)\left(\begin{array}{c}
J_{2}^{h} \\
\bar{g}_{2}^{h} \\
g_{2}^{h}
\end{array}\right)=\left(\begin{array}{c}
0 \\
-F \\
0
\end{array}\right) .
$$

The matrices $A, B, C$, and $D$ correspond to

$$
R_{K} \int_{K} \tau_{i} \cdot \tau_{j} d x, \quad \int_{K} \operatorname{div} \tau_{i} \phi_{j} d x, \quad \int_{K} \phi_{i} \phi_{j} d x, \quad \text { and } \int_{K} \tau_{i} \mu_{j} d x,
$$

respectively, where $\tau_{i}, \phi_{i}$, and $\mu_{i}$ are the basis functions of the corresponding spaces. The elementary matrices associated with the element $K$ will be denoted with the superscript $K$. Then it holds $\widetilde{B}^{K}=B^{K} S_{K} \in \mathbb{R}^{3}$. For $\widetilde{C}^{K}$ we get

$$
\widetilde{C}^{K}=C^{K} \operatorname{diag}\left(S_{e_{1}}, S_{e_{2}}, S_{e_{3}}\right) \text {. }
$$

In view of (26) and (25) the matrix $A$ has a diagonal structure and can easily be inverted, i.e. $J_{2}^{h}$ can be removed from the system by static condensation. Similar arguments for $-B^{T} A^{-1} \widetilde{B}-D$ allow to eliminate $\bar{g}_{2}^{h}$. This leads to a system only acting on $g_{2}^{h}$ :

$$
M g_{2}^{h}=G
$$

with

$$
\begin{aligned}
M & =C^{T} A^{-1} \widetilde{C}-C^{T} A^{-1} \widetilde{B}\left(B^{T} A^{-1} \widetilde{B}+D\right)^{-1} B^{T} A^{-1} \widetilde{C} \\
G & =C^{T} A^{-1} \widetilde{B}\left(B^{T} A^{-1} \widetilde{B}+D\right)^{-1} F .
\end{aligned}
$$

For the current density we get

$$
J_{2}^{h}=A^{-1}\left[\widetilde{B}\left(B^{T} A^{-1} \widetilde{B}+D\right)^{-1}\left(-F-B^{T} A^{-1} \widetilde{C} g_{2}^{h}\right)+\widetilde{C} g_{2}^{h}\right] .
$$


We make more precise the structure of the resulting matrix $M=\left(m_{i j}\right)$ on each triangle $K$ :

$$
m_{i j}^{K}= \begin{cases}\frac{S_{K}}{R_{K}} \frac{n_{1} \cdot n_{1}}{|K|}+\bar{c}_{2} \beta\left(\bar{c}_{2}\right) & \text { if } i=j=1, \\ \frac{S_{e_{j}(K)}}{R_{K}} \frac{n_{i} \cdot n_{j}}{|K|} & \text { else }\end{cases}
$$

where we have set $n_{i}=\left|e_{i}\right| \nu_{i}$. The function $\beta\left(\bar{c}_{2}\right)$, which is related to $C^{T} A^{-1} \widetilde{B}$ $\times\left(B^{T} A^{-1} \widetilde{B}+D\right)^{-1}$ and that scales the influences of the right-hand side and the zeroth-order term, is given by

$$
\beta\left(\bar{c}_{2}\right)=\left|e_{1}\right|^{2}|K|\left(\left|e_{1}\right|^{2}+\bar{c}_{2}|K|\left\|\tau_{3}\right\|_{L^{2}(K)}^{2} \frac{R_{K}}{S_{K}}\right)^{-1} .
$$

The coefficients of the right-hand side are:

$$
\gamma_{i}^{K}= \begin{cases}\beta\left(\bar{c}_{2}\right) \frac{1}{|K|} \int_{K}\left(-J_{1}^{h} \cdot \nabla V+\bar{c}_{1} \bar{g}_{1}\right) d x d y & \text { if } i=1, \\ 0 & \text { if } i=2,3 .\end{cases}
$$

For $\bar{c}_{2} \geq 0, \beta\left(\bar{c}_{2}\right)$ is a positive function. The quotient $S_{K} / R_{K}$ governs the strength of the convection and is strictly positive. Altogether we get an Mmatrix if the triangulation is weakly acute (see [30] for details).

Let $J_{2, i}^{K}$ be the nodal values of $J_{2}^{h}$ that correspond to the triangle $K$. From $g_{2}^{h}$ we reconstruct the current $J_{2}^{h}$ (with $g_{2, i}^{h}=\left.g_{2}^{h}\right|_{e_{i}}$ ):

$$
J_{2, i}^{K}= \begin{cases}\frac{1}{|K|} \sum_{j=1}^{3} S_{e_{j}(K)} R_{K}^{-1} g_{2, j}^{h}\left(n_{j}\right)_{i} & \text { if } i=1,2, \\ \left|e_{1}\right|^{-1}\left(-\gamma_{1}^{K}+\bar{c}_{2} \beta\left(\bar{c}_{2}\right)\right) & \text { if } i=3 .\end{cases}
$$

The approximation $J_{1}^{h}$ is constant on each triangle. With the same notation for $g_{1}^{h}$ we get

$$
\left.J_{1}^{h}\right|_{K}=\frac{1}{|K|} \sum_{j=1}^{3} S_{e_{j}(K)} R_{K}^{-1} g_{1, j}^{h} n_{j} .
$$

Remark 1 Since

$$
m_{i j}^{K}=\frac{n_{i} \cdot n_{j}}{|K|}, \quad i, j=1,2,3,
$$

is the elementary stiffness matrix corresponding to a $P_{1}$ non-conforming finiteelement discretization of the Laplace operator and since for constant potential it holds $S_{K} / R_{K}=1$, a treatment of the lower order terms in (22) with non-conforming $P_{1}$ elements is motivated. In the next chapter we perform a comparison of the different treatments. 


\subsection{Global Iteration}

For the classical drift-diffusion model, several iterative procedures for solving the coupled system have been proposed in the literature (see, e.g., [21, 28] and the references therein). We have used a Gummel-type method for the first numerical example, combined with an iteration procedure for the temperature, and a full Newton method for the second example. The Gummel iteration procedure can be regarded as an approximate Newton method, where the information about the strong coupling of the unknowns is incorporated into the Poisson equation and the Jacobian of the system is replaced by a diagonal matrix [21].

In the following we describe the Gummel-type iteration procedure. Before we give a description of the complete algorithm we explain the treatment of the temperature. From $g_{2}^{h}$ and $g_{1}^{h}$ we construct through (17) or (19) a temperature $T_{h}$ on each edge $e$. The local mean temperature $\left.\bar{T}\right|_{K}$ is then defined by the (arithmetic) mean value of $T_{h}$ on the edges $e \in \partial K$. This is different from the treatment in [13] since there $\left.\bar{T}\right|_{K}$ is constructed directly from $\bar{g}_{2}$ and $\bar{g}_{1}$. We observed that our method gives a slightly faster converging global iteration procedure (compared to the algorithm in [13]).

In the Poisson equation the electron density is defined through the equations (17) or (19). We assign the starting values $g_{1}^{(0)}, g_{2}^{(0)}$ (vectors of nodal values) and compute $V^{(0)}$ as the solution of the Poisson problem. Furthermore, assign three real numbers $\varepsilon, c, d>0$ and the vector $T^{(-1)}$. (For the values of $\varepsilon, c$, and $d$, see below.) Then, given $g_{1}^{(l)}, g_{2}^{(l)}$, and $V^{(l)}$, the iteration is defined as follows:

1. Compute the temperature $T^{(l)}$ as a function of $g_{1}^{(l)}, g_{2}^{(l)}$ (using the expressions (17) or (19)), set $V_{0}=V^{(l)}$ and define $\bar{T}$ (using $T^{(l)}$ ) as mentioned above.

2. (a) Find $g_{1}$ such that

$$
\begin{cases}-\operatorname{div} J_{1}=0, \quad J_{1}=\nabla g_{1}-\nabla V_{0} \bar{T}^{-1} g_{1} & \text { in } \Omega \\ g_{1}=g_{1}\left(n_{D}, T_{D}\right) & \text { on } \Gamma_{D} \\ J_{1} \cdot \nu=0 & \text { on } \Gamma_{N}\end{cases}
$$

(b) Set $V_{1}=V_{0}+\delta V$ where $\delta V$ is the solution of

$$
\left\{\begin{array}{l}
\lambda^{2} \Delta(\delta V)-n\left(g_{1}, T^{(l)}\right) \delta V=-\lambda^{2} \Delta V_{0}+n\left(g_{1}, T^{(l)}\right)-C \text { in } \Omega \\
\delta V=0 \text { on } \Gamma_{D}, \quad \nabla(\delta V) \cdot \nu=0 \quad \text { on } \Gamma_{N} .
\end{array}\right.
$$

If $\|\delta V\|_{L^{\infty}} \geq \max \left(\epsilon, c\left\|T^{(l-1)}-T^{(l)}\right\|^{d}\right)$ then set $V_{0}:=V_{1}$ and repeat step 2 , otherwise proceed with step 3 .

3. Set

$$
g_{1}^{(l+1)}=g_{1} \quad \text { and } \quad V^{(l+1 / 2)}=V_{1} .
$$


4. Find $g_{2}^{(l+1)}$ such that

$$
\begin{cases}-\operatorname{div} J_{2}^{(l+1)}+\bar{c}_{2} g_{2}^{(l+1)}=J_{1} \cdot \nabla V^{(l+1 / 2)}+\bar{c}_{1} g_{1}^{(l+1)} & \text { in } \Omega, \\ J_{2}^{(l+1)}=\nabla g_{2}^{(l+1)}-\nabla V^{(l+1 / 2)} \bar{T}^{-1} g_{2}^{(l+1)} & \text { in } \Omega, \\ g_{2}^{(l+1)}=g_{2}\left(n_{D}, T_{D}\right) \quad \text { on } \Gamma_{D}, \quad J_{2}^{(l+1)} \cdot \nu=0 & \text { on } \Gamma_{N} .\end{cases}
$$

5. Compute $T^{(l+1)}$ from $g_{1}^{(l+1)}, g_{2}^{(l+1)}$.

6. Define $V^{(l+1)}=V^{(l+1 / 2)}+\delta V$ where $\delta V$ is the solution of

$$
\left\{\begin{array}{l}
\lambda^{2} \Delta(\delta V)-n\left(g_{1}^{(l+1)}, T^{(l+1)}\right) \delta V \\
\quad=-\lambda^{2} \Delta V^{(l+1 / 2)}+n\left(g_{1}^{(l+1)}, T^{(l+1)}\right)-C \quad \text { in } \Omega \\
\delta V=0 \text { on } \Gamma_{D}, \quad \nabla(\delta V) \cdot \nu=0 \quad \text { on } \Gamma_{N} .
\end{array}\right.
$$

Steps 2 (a) and 4 are performed by means of the mixed exponential fitting scheme presented above (see (27)). Steps $2(\mathrm{~b})$ and 6 are solved via a $P_{1}$ nonconforming finite-element method (cf. [10]). After convergence of the iterative scheme, the current densities $J_{1}^{h}$ and $J_{2}^{h}$ are computed using formulas (33) and (34). We stress once more the fact that the use of the mixed formulation guarantees conservation of the current densities, i.e. the jump of the normal component of the current at the interelement boundaries is zero.

It is well known that a Gummel-type iteration scheme is very sensitive to the choice of initial guess, in particular far from thermal equilibrium. Therefore, the procedure is coupled to a continuation in the applied bias. For small bias steps or near the fixed point it is sufficent to perform steps 2(a) and (b) only once before proceeding to step 3. Performing this inner loop allows to use larger bias increments. However, it is not necessary to have convergence in the inner loop at every step for reaching convergence of the complete system. Altogether this is controlled by the truncation condition of step 2 . In the computations we have used the values $c=0.01, d=0.8$ and $\varepsilon=10^{-8}$.

\section{Numerical Examples}

We present two numerical tests. First we simulate a two-dimensional ballistic $n^{+} n n^{+}$silicon diode which is uniform in one space dimension. The numerical results are compared to the results of the one-dimensional ballistic diode simulated in [13]. In the second example we consider a two-dimensional silicon MESFET device. In Table 1 the physical constants we use here are collected.

\subsection{Ballistic Diode}

We consider a two-dimensional diode which is uniform in one dimension. The semiconductor domain is $\Omega=\left(0, l_{x}\right) \times\left(0, l_{y}\right)$, where $l_{x}=0.6 \mu \mathrm{m}, l_{y}=0.2 \mu \mathrm{m}$, and the length of the channel equals $0.4 \mu \mathrm{m}$. The $n^{+}$regions are defined in 


\begin{tabular}{|l|l|l|}
\hline Parameter & Physical meaning & Numerical value \\
\hline$q$ & the elementary charge & $1.6 \cdot 10^{-19} \mathrm{As}$ \\
$n_{i}$ & intrinsic density & $10^{10} \mathrm{~cm}^{-3}$ \\
$\varepsilon_{s}$ & permittivity constant & $10^{-12} \mathrm{AsV}^{-1} \mathrm{~s}^{-1}$ \\
$\mu_{0}$ & low field mobility & $1.5 \cdot 10^{3} \mathrm{~cm}^{2} \mathrm{~V}^{-1} \mathrm{~s}^{-1}$ \\
$U_{T}$ & thermal voltage at $T_{0}=300 \mathrm{~K}$ & $0.0259 \mathrm{~V}$ \\
$\tau_{0}$ & energy relaxation time & $0.4 \cdot 10^{-12} \mathrm{~S}$ \\
\hline
\end{tabular}

Table 1: Physical parameters for silicon.

$(0,0.1 \mu \mathrm{m}) \times\left(0, l_{y}\right)$ and $\left(0.5 \mu \mathrm{m}, l_{x}\right) \times\left(0, l_{y}\right)$, the $n$ region (or channel region) in $(0.1 \mu \mathrm{m}, 0.5 \mu \mathrm{m}) \times\left(0, l_{y}\right)$. The diode has two Ohmic contacts on both sides of the domain whereas the remaining boundary parts are insulating. We choose:

$$
\Gamma_{D 1}=\{x=0\}, \quad \Gamma_{D 2}=\left\{x=l_{x}\right\}, \quad \Gamma_{N}=\{y=0\} \cup\left\{y=l_{y}\right\} .
$$

On $\Gamma_{N}$ homogeneous Neumann boundary data are imposed and on $\Gamma_{D}$ the Dirichlet boundary data are given as follows:

$$
\begin{aligned}
& n=C_{m}, \quad T=T_{0}, \quad V=\Phi_{0} \quad \text { on } \Gamma_{D 1}, \\
& n=C_{m}, \quad T=T_{0}, \quad V=U+\Phi_{0} \quad \text { on } \Gamma_{D 2} \text {. }
\end{aligned}
$$

Here, the ambient temperature is $T_{0}=300 \mathrm{~K}, U=1.5 \mathrm{~V}$ is the applied voltage, and the built-in potential $\Phi_{0}$ is given by

$$
\Phi_{0}=U_{T} \ln \left(n / n_{i}\right) .
$$

The doping concentration in the $n^{+}$region is $C_{m}=5 \cdot 10^{17} \mathrm{~cm}^{-3}$, in the channel region we take a doping density of $2 \cdot 10^{15} \mathrm{~cm}^{-3}$. These values are the same as in $[7,13,32,36]$ and allow for a comparison with the results presented in these papers.

First we report the relative errors (RE) of the computed solutions in the $L^{2}$ norm in Table 2 and Table 3, using the Chen model (see Section 2). The mesh on which the reference solution was computed has 20480 triangles and maximal edge length $h=1 / 160$. As mentioned in Remark 1 we compare the proposed scheme with a $P_{1}$ non-conforming treatment of the zeroth-order terms. The convergence rates of the mixed discretization for $T_{h}, n_{h}, g_{2, h}$ are 1.55, 1.85, and 1.84, respectively. For a constant potential one would expect the $P_{1}$ nonconforming scheme to give better results, since there the lower-order terms have a stronger effect on the resulting matrix. For the present situation the errors do almost not differ. This shows that the convection parts play here an important role. We finally mention that similar results have been obtained using the Lyumkis model.

Now we present the numerical results for a non-uniform mesh with 900 triangles. In Figure 2 the (unscaled) electron temperature for the Lyumkis and the 


\begin{tabular}{|c|c|c|c|c|}
\hline$h$ & RE for $\Psi_{h}$ & RE for $T_{h}$ & RE for $n_{h}$ & RE for $g_{2, h}$ \\
\hline \hline 0.1 & $5.4 \cdot 10^{-3}$ & 0.0122 & 0.100 & 0.100 \\
\hline 0.05 & $1.9 \cdot 10^{-3}$ & $4.96 \cdot 10^{-3}$ & 0.029 & 0.029 \\
\hline 0.025 & $5.1 \cdot 10^{-4}$ & $1.4 \cdot 10^{-3}$ & $7.7 \cdot 10^{-3}$ & $7.7 \cdot 10^{-3}$ \\
\hline
\end{tabular}

Table 2: Relative errors for the mixed scheme (Chen model).

\begin{tabular}{|c|c|c|c|c|}
\hline$h$ & RE for $\Psi_{h}$ & RE for $T_{h}$ & RE for $n_{h}$ & RE for $g_{2, h}$ \\
\hline \hline 0.1 & $5.4 \cdot 10^{-3}$ & 0.0122 & 0.100 & 0.100 \\
\hline 0.05 & $1.9 \cdot 10^{-3}$ & $3.4 \cdot 10^{-3}$ & 0.029 & 0.029 \\
\hline 0.025 & $4.8 \cdot 10^{-4}$ & $9.9 \cdot 10^{-4}$ & $7.8 \cdot 10^{-3}$ & $7.8 \cdot 10^{-3}$ \\
\hline
\end{tabular}

Table 3: Relative errors for the $P_{1}$ non-conforming scheme (Chen model).

Chen model are shown. As expected, the temperature profiles are almost uniform in one space direction. Moreover, they coincide with the values computed in [13].

In Figure 3 we show the (unscaled) electron mean velocity $u=J_{1} /(q n)$. Again, the profiles coincide almost with the corresponding results in [13]. The maximum values for the Lyumkis and the Chen model are $3.03 \cdot 10^{7} \mathrm{~cm} / \mathrm{s}$ and $1.44 \cdot 10^{7} \mathrm{~cm} / \mathrm{s}$, respectively. In [13], the maximum values $2.92 \cdot 10^{7} \mathrm{~cm} / \mathrm{s}$ (Lyumkis model) and $1.44 \cdot 10^{7} \mathrm{~cm} / \mathrm{s}$ (Chen model) are reported.

\subsection{MESFET Device}

The MESFET (metal-semiconductor field-effect transistor) device is used as a switch or amplifier [38]. The device geometry is as follows. The device consists of two highly-doped $n^{+}$regions near the Ohmic contacts (called source and drain) and an $n$ region with a Schottky contact (called gate); see Figure 4 . The source and drain contact lengths are $0.1 \mu \mathrm{m}$; the gate contact length is $0.2 \mu \mathrm{m}$. The complete geometry is shown in Figure 4. For the doping profile we use the smoothed function presented in Figure 5. It holds (in $\mu \mathrm{m}$ ):

$C(x, y)= \begin{cases}3 \cdot 10^{17} \mathrm{~cm}^{-3} & :(x, y) \in[0,0.1] \times[0.15,0.2] \cup[0.5,0.6] \times[0.15,0.2] \\ 1 \cdot 10^{17} \mathrm{~cm}^{-3} & : \text { else }\end{cases}$

The boundary data is given as follows (with $\Phi_{0}$ as defined in (39)):

- at the source: $n=3 \cdot 10^{17} \mathrm{~cm}^{-3}, T=300 \mathrm{~K}, V=\Phi_{0}$;

- at the drain: $n=3 \cdot 10^{17} \mathrm{~cm}^{-3}, T=300 \mathrm{~K}, V=\Phi_{0}+2 \mathrm{~V}$;

- at the gate: $n=3.9 \cdot 10^{5} \mathrm{~cm}^{-3}, T=300 \mathrm{~K}, V=\Phi_{0}-0.8 \mathrm{~V}$; 


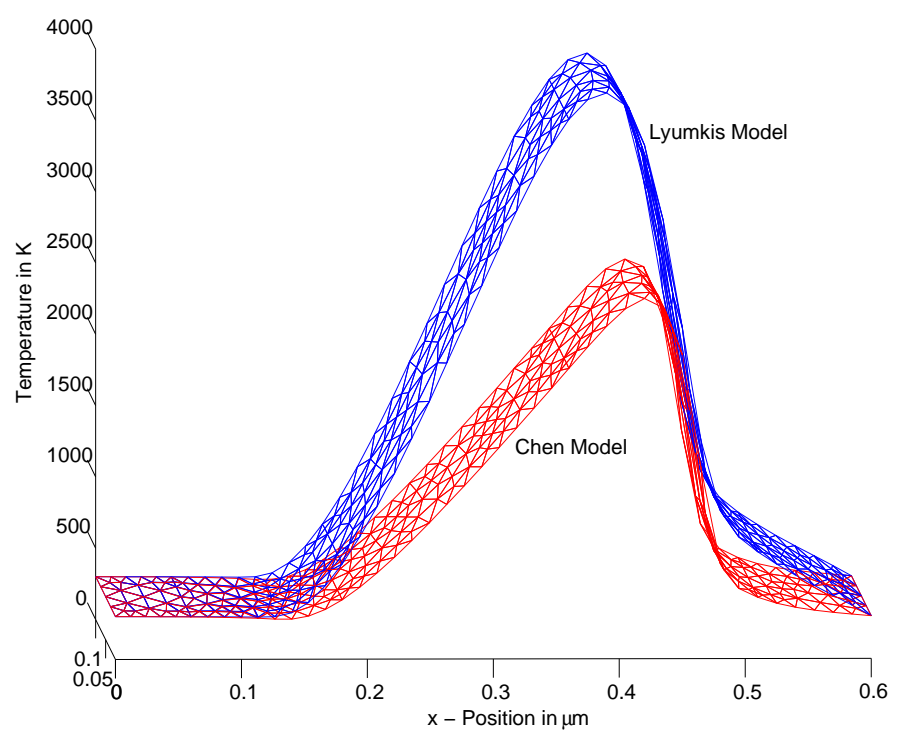

Figure 2: Electron temperature versus position in a ballistic diode.

- for the remaining boundary segments, homogeneous Neumann boundary conditions for $J_{1}, J_{2}$, and $V$ are used.

These values are the same as in [9,23], but we do not prescribe the velocity on the contact parts. The value for $n$ at the gate contact has been computed from the formula (5.1-19) from [35]. We use the definition of the temperature and the energy relaxation term of the Chen model.

For this device geometry, the Gummel-type iteration presented in Section 3.2 converges very slowly. The reason may be the complex structure of the temperature profile, in particular the large gradients near the gate contact. For this reason, a full Newton scheme has been used.

In the Newton algorithm, the discrete algebraic equations for $T=f\left(g_{1}, g_{2}\right)$ (see (17)) must be properly scaled in order to avoid a badly conditioned Jacobian matrix. We assume that $T_{h}$ and $f_{h}$, given by $T_{h}=f_{h}=f\left(g_{1}^{h}, g_{2}^{h}\right)$, are a $P_{1}$ non-conforming approximation of the temperature and the right-hand side of (17) and the equation $T_{h}=f_{h}$ is considered in variational form as follows:

$$
\int_{\Omega} T_{h j} \varphi_{j} \varphi_{i} d x d y=\int_{\Omega} f_{h j} \varphi_{j} \varphi_{i} d x d y
$$

where $\varphi_{i}$ denotes the basis function. Now, since $\int \varphi_{j} \varphi_{i} d x d y=\left|\operatorname{supp}\left(\varphi_{j}\right)\right| / 3 \cdot \delta_{i j}$ we get

$$
T_{h j}\left|\operatorname{supp}\left(\varphi_{j}\right)\right| / 3=f_{h j}\left|\operatorname{supp}\left(\varphi_{j}\right)\right| / 3
$$




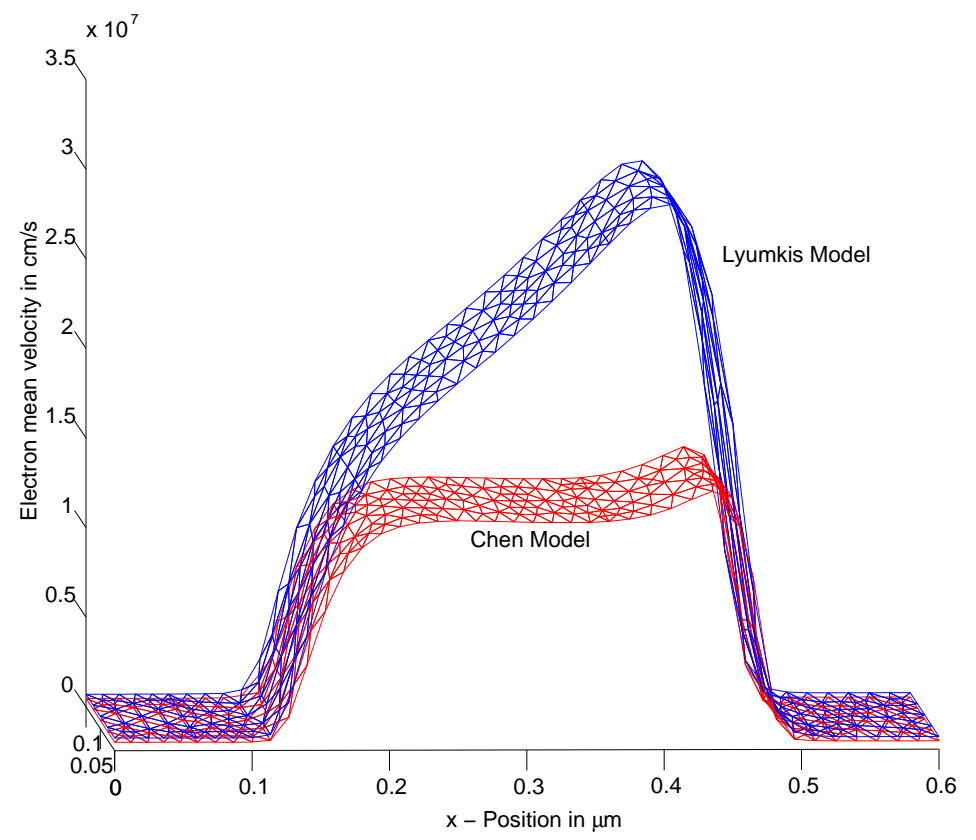

Figure 3: Electron mean velocity versus position in a ballistic diode.

which is of the same order of magnitude as the zeroth-order term in the differential equations. This leads to much faster convergence especially for bad initial values or high increments in the bias continuation.

Figures 6-9 show the computational results for a mesh with 1564 nodes. It is only refined near the Dirichlet boundaries and the junctions. In Figure 6 the electron density is shown. The depletion area around the gate contact is clearly seen.

The electron temperature is presented in Figure 7. The maximal temperature is $3152 \mathrm{~K}$. As expected, the temperature is large near the drain contact. Near the gate, the gradient of the temperature is very large, which may indicate that the fixed boundary temperature of $300 \mathrm{~K}$ is physically not appropriate.

In Figure 8 the electrostatic potential is shown. Since the electrons are moving in the direction of positive potential, we expect a significant current flow from the source to the drain. This is confirmed by Figure 9 where the vector of the particle current density is depicted. In fact, with the above data, the MESFET device is in an "open" state. In a "closed" state the depletion region is much larger than shown in Figure 6 and the current density much smaller. 


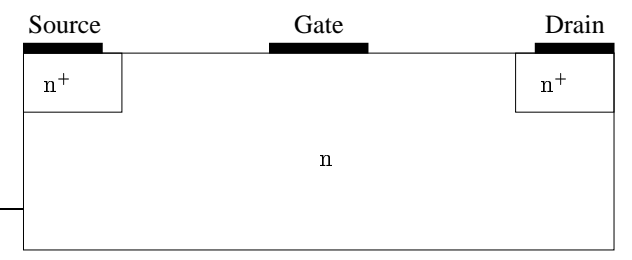

Figure 4: Geometry of the MESFET.

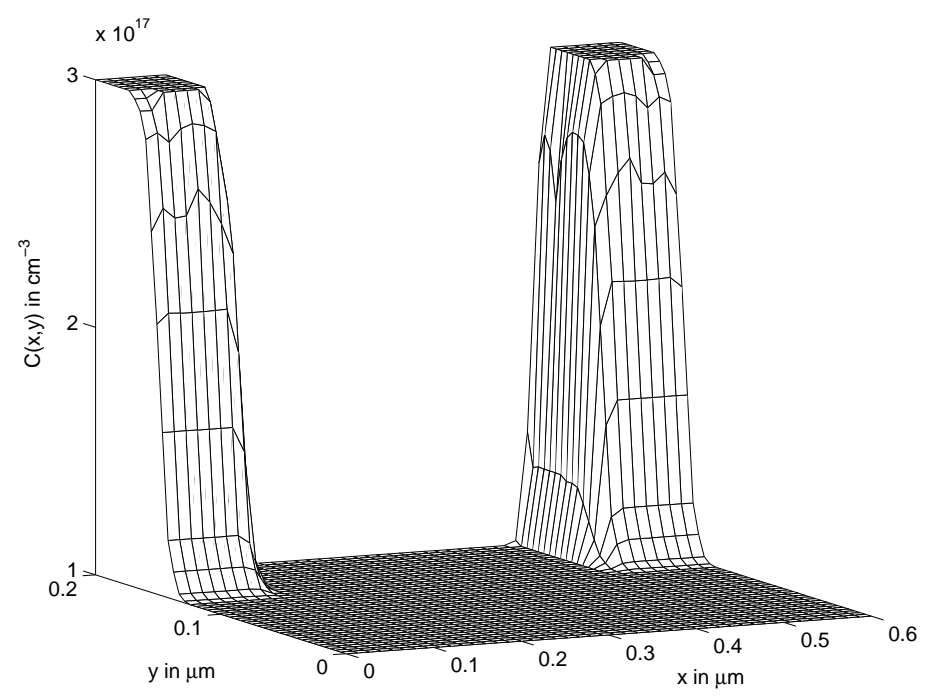

Figure 5: Doping profile of the MESFET.

\section{Conclusion}

In this paper we have adopted the mixed finite elements developed in [30] to the two-dimensional energy-transport equations. The three main features of the numerical scheme are that the current densities are conserved (i.e. the jump of the normal component of the current at the interelement boundaries is zero), that the resulting stiffness matrix is an M-matrix (which guarantees positivity of the electron density and provides robustness of the scheme in regions with very low carrier concentrations), and that the convection part is approximated with the correct order of magnitude even in the presence of zeroth-order terms.

We have presented numerical results for a ballistic diode and a MESFET device. Due to the advantages of the employed scheme, rather coarse meshes could be used. Some care has to be taken on the discrete temperature in regions where it varies strongly.

The boundary layer of the temperature near the gate contact indicates that 


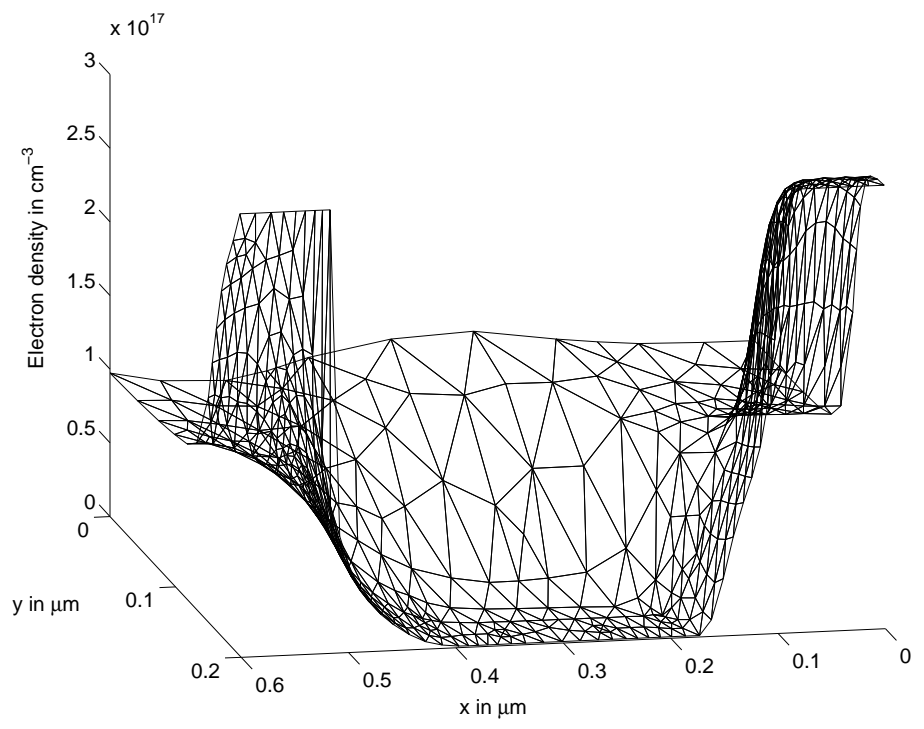

Figure 6: Electron density in the MESFET (Chen model).

different boundary conditions may be physically more appropriate. One possibility is to employ Robin boundary conditions at the contacts, since they are second-order approximations of boundary conditions for the Boltzmann equation [14] and the energy-transport equations are itself derived from the Boltzmann equation [3]. Robin boundary conditions have been already used in the drift-diffusion model [41]. This investigation will be done in a future work.

\section{References}

[1] W. Allegretto and H. Xie. Nonisothermal semiconductor systems. In X. Liu and D. Siegel, editors, Comparison methods and stability theory, volume 162 of Lecture Notes in Pure and Applied Mathematics, New York, 1994. Marcel Dekker.

[2] Y. Apanovich, P. Blakey, R. Cottle, E. Lyumkis, B. Polsky, A. Shur, and A. Tcherniaev. Numerical simulations of submicrometer devices including coupled nonlocal transport and nonisothermal effects. IEEE Trans. El. Dev., 42:890-897, 1995.

[3] N. Ben Abdallah and P. Degond. On a hierarchy of macroscopic models for semiconductors. J. Math. Phys., 37:3308-3333, 1996.

[4] K. Bløtekjær. Transport equations for electrons in two-valley semiconductors. IEEE Trans. El. Dev., 17:38-47, 1970.

[5] F. Brezzi, L. Marini, and P. Pietra. Numerical simulation of semiconductor devices. Comp. Meth. Appl. Mech. Engrg., 75:493-514, 1989.

[6] F. Brezzi, L. Marini, and P. Pietra. Two-dimensional exponential fitting and applications to drift-diffusion models. SIAM J. Num. Anal., 26:1342-1355, 1989. 


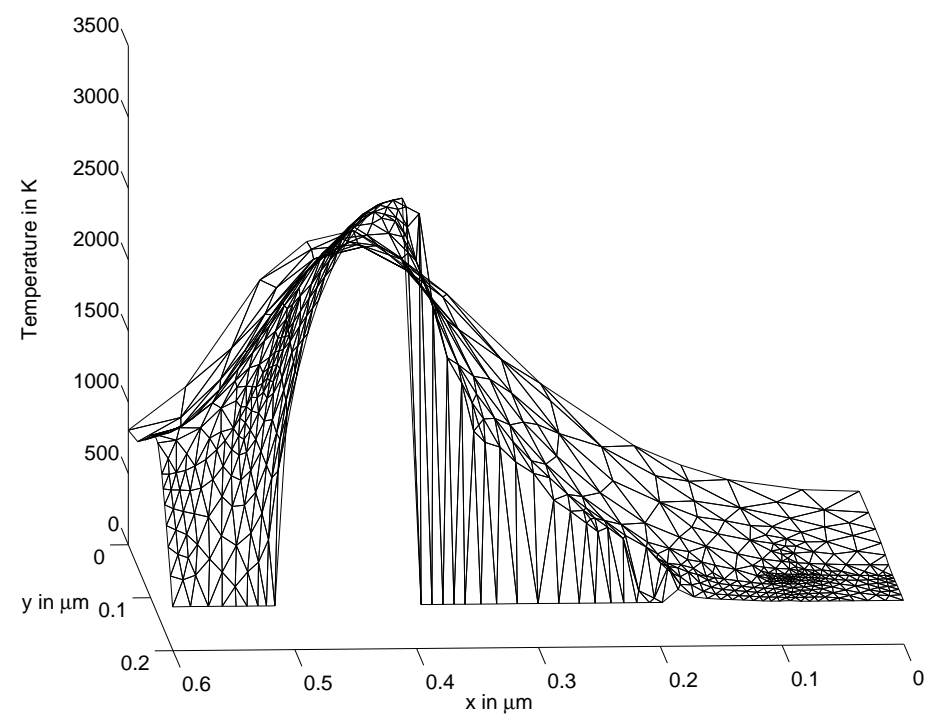

Figure 7: Electron temperature in the MESFET (Chen model).

[7] D. Chen, E. Kan, U. Ravaioli, C. Shu, and R. Dutton. An improved energy transport model including nonparabolicity and non-Maxwellian distribution effects. IEEE Electr. Dev. Letters, 13:26-28, 1992.

[8] D. Chen, E. Sangiorgi, M. Pinto, E. Kan, U. Ravaioli, and R. Dutton. Analysis of spurious velocity overshoot in hydrodynamic simulations. NUPAD IV, pages 109-114, 1992.

[9] Z. Chen, B. Cockburn, J. Jerome, and C. Shu. Finite element computation of the hydrodynamic model of semiconductor devices. VLSI Design, 3:145-158, 1995.

[10] M. Crouzeix and P.A. Raviart. Conforming and nonconforming finite element methods for solving the stationary Stokes equation. RAIRO, 7:33-76, 1973.

[11] P. Degond, S. Génieys, and A. Jüngel. A system of parabolic equations in nonequilibrium thermodynamics including thermal and electrical effects. J. Math. Pures Appl., 76:991-1015, 1997.

[12] P. Degond, S. Génieys, and A. Jüngel. A steady-state system in nonequilibrium thermodynamics including thermal and electrical effects. Math. Meth. Appl. Sci., 21:1399-1413, 1998.

[13] P. Degond, A. Jüngel, and P. Pietra. Numerical discretization of energy-transport model for semiconductors with non-parabolic band structure. SIAM J. Sci. Comp., 22:986-1007, 2000.

[14] P. Degond and C. Schmeiser. Kinetic boundary layers and fluid-kinetic coupling in semiconductors. Transp. Theory Stat. Phys., 28:31-55, 1999.

[15] W. Fang and K. Ito. Existence of stationary solutions to an energy drift-diffusion model for semiconductor devices. Math. Models Meth. Appl. Sci., 11:827-840, 2001. 


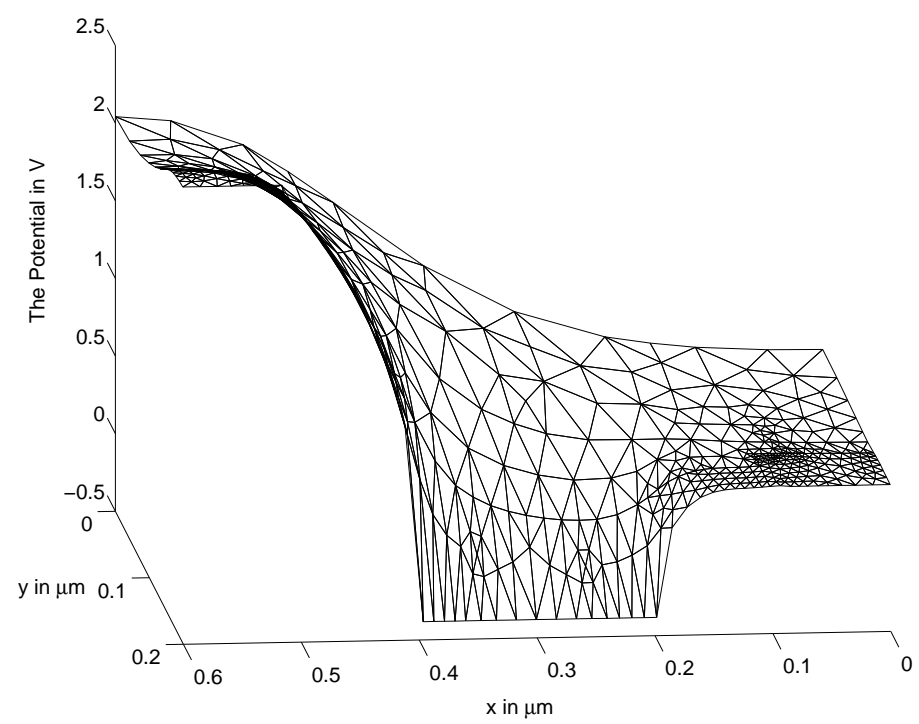

Figure 8: Electrostatic potential in the MESFET (Chen model).

[16] M. Fournié. Construction et analyse de schémas compacts d'ordre élevé pour des problèmes fortement convectifs. Application à la simulation de semi-conducteurs. PhD thesis, Université Paul Sabatier, Toulouse, France, 1999.

[17] C. Gardner, J. Jerome, and D. Rose. Numerical methods for the hydrodynamic device model: subsonic flow. IEEE Trans. CAD, 8:501-507, 1989.

[18] I. Gasser and R. Natalini. The energy-transport and the drift-diffusion equations as relaxation limits of the hydrodynamic model for semiconductors. Quart. Appl. Math., 57:269-282, 1999.

[19] J. Griepentrog. An application of the implicit function theorem to an energy model of the semiconductor theory. Z. Angew. Math. Mech., 79:43-51, 1999.

[20] J. Jerome. The approximation problem for drift-diffusion systems. SIAM Review, $37: 552-572,1995$.

[21] J. Jerome. Analysis of charge transport. A mathematical study of semiconductor devices. Springer, Berlin, 1996.

[22] J. Jerome and T. Kerkhoven. A finite-element approximation theory for the driftdiffusion semiconductor model. SIAM J. Num. Anal., 28:403-422, 1991.

[23] J. Jerome and C.-W. Shu. Energy models for one-carrier transport in semiconductor devices. In W. Coughran, J. Colde, P. Lloyd, and J. White, editors, Semiconductors, Part II, volume 59 of IMA Volumes in Mathematics and its Applications, pages 185-207, New York, 1994. Springer.

[24] J. Jerome and C.-W. Shu. Energy transport systems for semiconductors: Analysis and simulation. In V. Lakshmikantham, editor, Proccedings of the First World Congress of Nonlinear Analysts, pages 3835-3846, Berlin, 1996. Walter de Gruyter. 


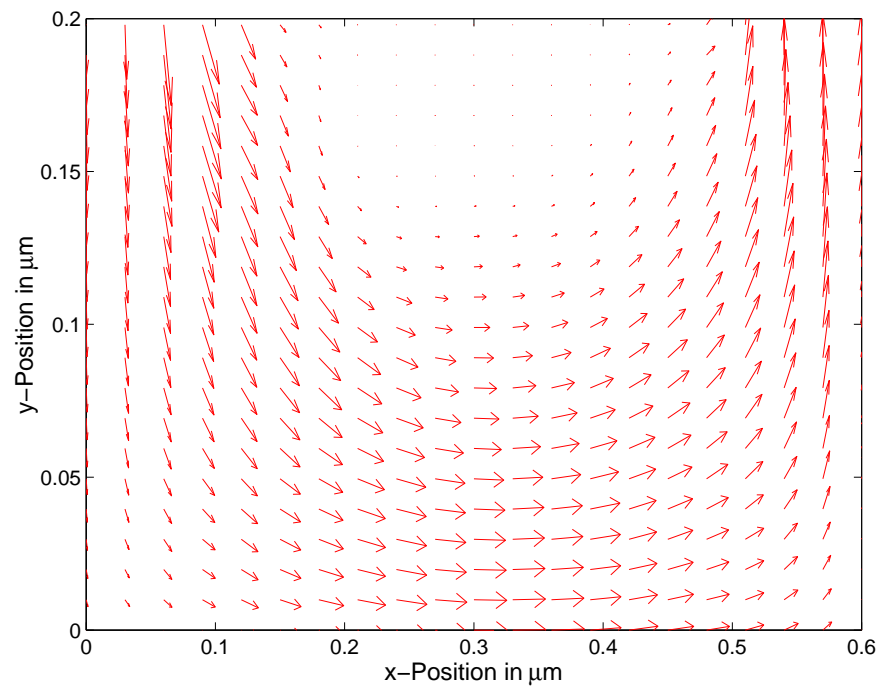

Figure 9: Electron current density in the MESFET (Chen model).

[25] A. Jüngel. Quasi-hydrodynamic Semiconductor Equations. Progress in Nonlinear Differential Equations. Birkhäuser, Basel, 2001.

[26] A. Jüngel and P. Pietra. A discretization scheme of a quasi-hydrodynamic semiconductor model. Math. Models Meth. Appl. Sci., 7:935-955, 1997.

[27] E. Lyumkis, B. Polsky, A. Shur, and P. Visocky. Transient semiconductor device simulation including energy balance equation. Compel, 11:311-325, 1992.

[28] T. Kerkhoven and Y. Saad. On acceleration methods for coupled nonlinear elliptic systems. Numer. Math., 60:525-548, 1992.

[29] L. D. Marini and P. Pietra. An abstract theory for mixed approximations of second order elliptic equations. Mat. Aplic. Comp., 8:219-239, 1989.

[30] L. D. Marini and P. Pietra. New mixed finite element schemes for current continuity equations. COMPEL, 9:257-268, 1990.

[31] P. A. Markowich, C. A. Ringhofer, and C. Schmeiser. Semiconductor Equations. Springer, 1990.

[32] A. Marrocco, P. Montarnal, and B. Perthame. Simulation of the energy-transport and simplified hydrodynamic models for semiconductor devices using mixed finite elements. In Proceedings ECCOMAS 96, London, 1996. John Wiley.

[33] P. Raviart and J. Thomas. A mixed finite element method for second order elliptic equations. In Mathematical Aspects of the Finite Element Method, volume 606 of Lecture Notes in Math., pages 292-315. Springer, 1977.

[34] M. Rudan, A. Gnudi, and W. Quade. A generalized approach to the hydrodynamic model of semiconductor equations. In G. Baccarani, editor, Process and Device Modeling for Microelectronics, Amsterdam, 1993. Elsevier. 
[35] S. Selberherr. Analysis and Simulation of Semiconductor Devices. Springer, Wien, 1984.

[36] K. Souissi, F. Odeh, H. Tang, and A. Gnudi. Comparative studies of hydrodynamic and energy transport models. COMPEL, 13:439-453, 1994.

[37] R. Stratton. Diffusion of hot and cold electrons in semiconductor barriers. Phys. Rev., 126:2002-2014, 1962.

[38] S. Sze. Physics of Semiconductor Devices. John Wiley, New York, 1981.

[39] P. Visocky. A method for transient semiconductor device simulation using hotelectron transport equations. In J. Miller, editor, Proc. of the Nasecode X Conf., Dublin, 1994. Boole Press.

[40] W. Walus. Computational methods for the Boltzmann equation. In N. Bellomo, editor, Lecture Notes on the Mathematical Theory of the Boltzmann Equation, pages 179-223. World Scientific, Singapore, 1995.

[41] A. Yamnahakki. Second order boundary conditions for the drift-diffusion equations of semiconductors. Math. Models Meth. Appl. Sci., 5:429-455, 1995. 